Supporting information for

\title{
Crystal Growth, THz Generation, and Optical Characterization of EHPSI-4NBS
}

Gabriel A. Valdivia-Berroeta, Isaac C. Tangen, Charles B. Bahr, Karissa C. Kenney, Erika W. Jackson, Jess DeLagange, David J. Michaelis*, and Jeremy A. Johnson*

Department of Chemistry and Biochemistry, Brigham Young University, Provo, UT 84602

Corresponding author email: dmichaelis@chem.byu.edu; jjohnson@chem.byu.edu

Figure S1. UV-NIR transparency spectra of EHPSI-4NBS and BNA.

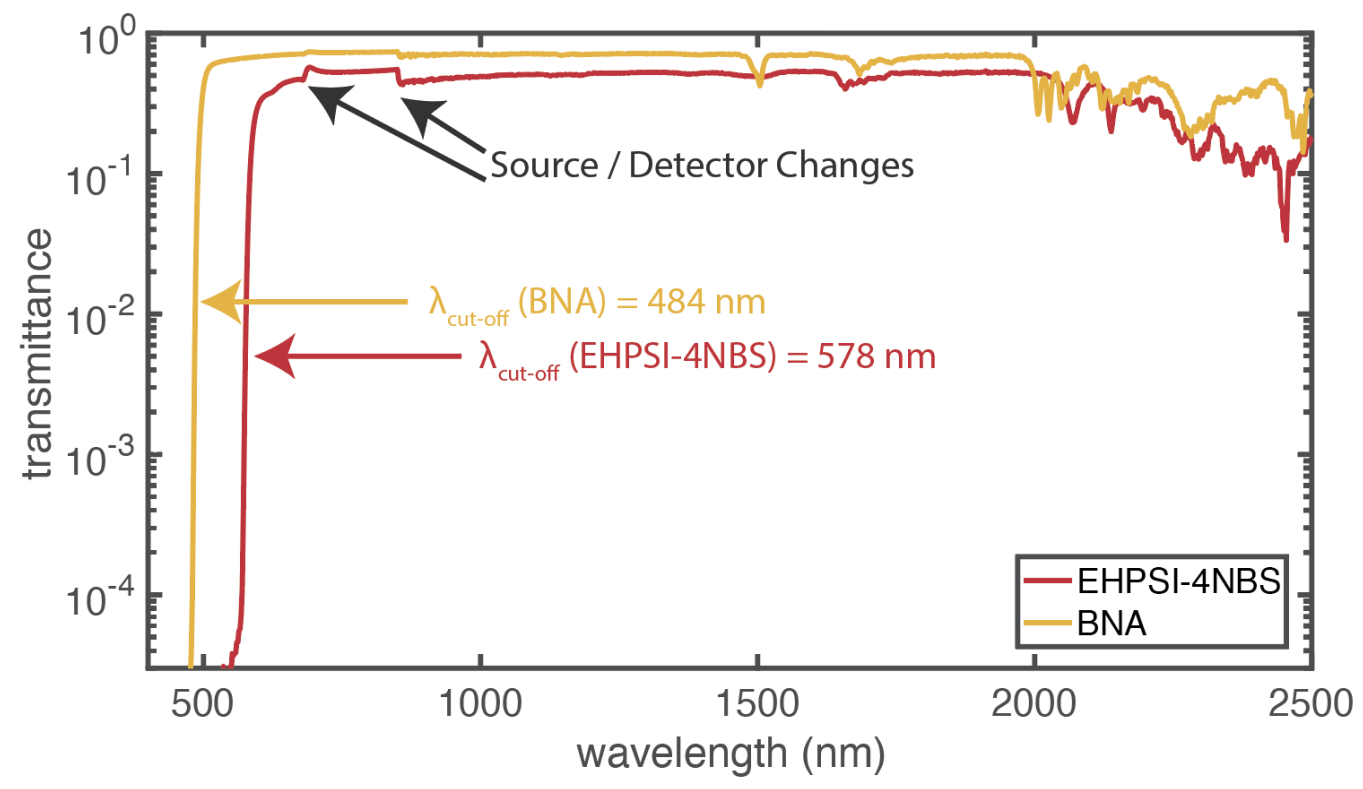

